\title{
Test of $\bar{N} N$ potential models: Isospin relations in $\bar{p} d$ annihilations at rest and the search for quasinuclear bound states
}

\author{
A. Abele ${ }^{8}$, J. Adomeit ${ }^{7}$, C. Amsler ${ }^{16}$, C.A. Baker ${ }^{5}$, B.M. Barnett ${ }^{3}$, C.J. Batty ${ }^{5}$, M. Benayoun ${ }^{13}$, A. Berdoz $^{14}$, \\ K. Beuchert ${ }^{2}$, S. Bischoff ${ }^{8}$, P. Blüm ${ }^{8}$, K. Braune ${ }^{12}$, J. Brose ${ }^{11}$, D.V. Bugg ${ }^{9}$, T. Case ${ }^{1}$, O. Cramer ${ }^{12}$, V. Credé ${ }^{3}$ \\ K.M. Crowe ${ }^{1}$, T. Degener ${ }^{2}$, N. Djaoshvili ${ }^{12}$, S. v. Dombrowski ${ }^{16}$, M. Doser ${ }^{6}$, W. Dünnweber ${ }^{12}$, D. Engelhardt ${ }^{8}$, \\ M.A. Faessler ${ }^{12}$, P. Giarritta ${ }^{16}$, R.P. Haddock ${ }^{10}$, F.H. Heinsius ${ }^{1}$, M. Heinzelmann ${ }^{16}$, A. Herbstrith ${ }^{8}$, M. Herz ${ }^{3}$, \\ N.P. Hessey ${ }^{12}$, P. Hidas ${ }^{4}$, C. Hodd ${ }^{9}$, C. Holtzhaußen ${ }^{8}$, D. Jamnik ${ }^{12}$, H. Kalinowsky ${ }^{3}$, B. Kämmle ${ }^{7}$, P. Kammel ${ }^{1}$, \\ J. Kisiel ${ }^{6}$, E. Klempt ${ }^{3}$, H. Koch ${ }^{2}$, C. Kolo ${ }^{12}$, M. Kunze ${ }^{2}$, U. Kurilla ${ }^{2}$, M. Lakata ${ }^{1}$, R. Landua ${ }^{6}$, H. Matthäy ${ }^{2}$, \\ R. McCrady ${ }^{14}$, J. Meier ${ }^{7}$, C.A. Meyer ${ }^{14}$, L. Montanet ${ }^{6}$, R. Ouared ${ }^{6}$, F. Ould-Saada ${ }^{16}$, K. Peters ${ }^{2}$, B. Pick $^{3}$, \\ C. Pietra ${ }^{16}$, C.N. Pinder ${ }^{5}$, M. Ratajczak ${ }^{2}$, C. Regenfus ${ }^{12}$, S. Resag ${ }^{3}$, W. Roethel ${ }^{12}$, P. Schmidt ${ }^{7}$, I. Scott ${ }^{9}$, R. Seibert ${ }^{7}$, \\ S. Spanier ${ }^{16}$, H. Stöck ${ }^{2}$, C. Straßburger ${ }^{3}$, U. Strohbusch ${ }^{7}$, M. Suffert ${ }^{15}$, J.S. Suh ${ }^{3}$, U. Thoma ${ }^{3}$, M. Tischhäuser ${ }^{8}$, \\ I. Uman ${ }^{12}$, C. Völcker ${ }^{12}$, S. Wallis ${ }^{12}$, D. Walther ${ }^{2}$, U. Wiedner ${ }^{6}$, K. Wittmack ${ }^{3}$, B.S. Zou ${ }^{9}$, and C. Zupančič ${ }^{12}$ \\ 1 University of California, LBNL, Berkeley, CA 94720, USA \\ 2 Universität Bochum, D-44780 Bochum, FRG \\ 3 Universität Bonn, D-53115 Bonn, FRG \\ 4 Academy of Science, H-1525 Budapest, Hungary \\ 5 Rutherford Appleton Laboratory, Chilton, DidcotOX110QX, UK \\ 6 CERN, CH-1211 Geneva 4, Switzerland \\ 7 Universität Hamburg, D-22761 Hamburg, FRG \\ 8 Universität Karlsruhe, D-76021 Karlsruhe, FRG \\ ${ }^{9}$ Queen Mary and Westfield College, London E1 4NS, UK \\ 10 University of California, Los Angeles, CA 90024, USA \\ 11 Universität Mainz, D-55099 Mainz, FRG \\ 12 Universität München, D-80333 München, FRG \\ 13 LPNHE Paris VI, VII, F-75252 Paris, France \\ 14 Carnegie Mellon University, Pittsburgh, PA 15213, USA \\ 15 Centre de Recherches Nucléaires,F-67037 Strasbourg, France \\ 16 Universität Zürich, CH-8057 Zürich,Switzerland
}

December 3, 1999

\begin{abstract}
We have determined branching ratios for antiproton annihilations at rest on protons or neutrons in liquid deuterium which we compare to frequencies of isospin-related processes in antiproton-proton annihilations. Using the annihilation rates into $\pi^{0} \pi^{0}$ and $\pi^{-} \pi^{0}$ where the annihilation took place on the proton or neutron, respectively, we discuss the fraction of S-wave and P-wave annihilation in liquid $\mathrm{D}_{2}$. The frequencies for $\pi^{-} \omega$ and $\rho^{-} \pi^{0}$, and $\pi^{-} \eta$ and $\pi^{-} \eta^{\prime}$ and the corresponding frequencies for $\bar{p} p$ annihilations are used to determine isoscalar and isovector contributions to the protonium wave function. The isospin decomposition of the annihilating $\bar{p} p$ system in the ${ }^{3} \mathrm{~S}_{1}$ or ${ }^{1} \mathrm{~S}_{0}$ state is consistent with both, pure $\bar{p} p$ initial wave function and with the predictions of $\overline{\mathrm{N}} \mathrm{N}$ potential models. For the ${ }^{3} \mathrm{P}_{0}$ state of the $\bar{p}$ atom we find consistency with a pure $\bar{p}$ system at annihilation while $\bar{N} N$ potential models predict large $\bar{n} n$ contributions.

We observe $\rho$ - $\omega$ interference in $\overline{\mathrm{p}} \rightarrow \pi^{+} \pi^{-} \eta$ and $\pi^{+} \pi^{-} \pi^{0}$ annihilation which we compare to $\rho$ - $\omega$ interference in $e^{+} e^{-}$annihilation. The interference patterns show striking similarities due to similar phase relations; the interference magnitude depends on the $\omega$ - $\rho$ production ratio. The similarity of the phase in all 3 data sets demonstrates that isovector and isoscalar parts of the protonium ( $\overline{\mathrm{p}} \mathrm{p}$ atomic) wave function are relatively real, again in conflict with $\overline{\mathrm{N} N}$ potential models.

The annihilation rate for $\overline{\mathrm{p}} \mathrm{d} \rightarrow \mathrm{K}^{-} \mathrm{K}^{0} \mathrm{p}$ confirms the dominance of the isovector contribution to $\overline{\mathrm{N}} \mathrm{N} \rightarrow \mathrm{K} \overline{\mathrm{K}}$ annihilations. No complications due to initial state interactions are required by the data. Furthermore, we searched for narrow quasinuclear bound states close to the $\overline{\mathrm{N}} \mathrm{N}$ threshold, also predicted by $\overline{\mathrm{N}} \mathrm{N}$ potential models, but with negative outcome. We conclude that $\overline{\mathrm{N}} \mathrm{N}$ potential models are not suited to provide insight into the dynamics of the annihilation process.
\end{abstract}




\section{Introduction}

It is well known that antinucleon-nucleon interactions are intimately related to nucleon-nucleon interactions [1]. The same mesons responsible for NN interactions also create $\overline{\mathrm{N}} \mathrm{N}$ interactions, the only difference is a sign change of the interaction potential when the G-parity of the exchanged meson is negative. The role of $\pi$ exchange was demonstrated explicitly at LEAR [2]; even the $\mathrm{N} \pi$ coupling constant could be derived from $\overline{\mathrm{p}} \mathrm{p} \rightarrow \overline{\mathrm{n}} \mathrm{n}$ charge exchange scattering. Thus the concept of meson exchange as input to models of $\overline{\mathrm{N} N}$ interactions seems to rest on healthy foundations [3].

Yet $\overline{\mathrm{N}} \mathrm{N}$ potential models make a number of further predictions which can be tested experimentally. Two of them concern strong interaction effects in the protonium $(\overline{\mathrm{p}} \mathrm{p})$ atom. Strong interaction shifts and widths had been predicted [4] long before the quantities were determined experimentally [5]. Excellent agreement between model predictions and experimental results was observed. However, $\overline{\mathrm{N} N}$ potential models also predict that initial state interactions in the $\bar{p} p$ atom lead to a significant isospin distortion of the wave function, to a sizable $\bar{n} n$ component in the protonium wave function [6]. Experimentally, this effect was confirmed but only with 1-standard-deviation significance [7].

The most striking consequence of $\overline{\mathrm{N}} \mathrm{N}$ potential models was the prediction of long-lived $\overline{\mathrm{N}} \mathrm{N}$ states. These quasinuclear (deuteron like) states are a direct consequence of the reversal of the repulsive core in $\mathrm{NN}$ interactions into very strong attraction in the $\overline{\mathrm{N}} \mathrm{N}$ system $[8,9]$. We now know that narrow quasinuclear bound states do not exist [10]. Yet there might exist broad quasinuclear $\overline{\mathrm{N} N}$ states. The $f_{2}(1565)$, also known as $\operatorname{AX}(1565)$ [11], which does not fit well into any $\bar{q} q$ nonet has been interpreted as such a state [12]. If this is indeed the case, its width of $170 \mathrm{MeV}$ would predict rather larger widths for most $\overline{\mathrm{N}} \mathrm{N}$ bound states. Only loosely bound states close to the $\overline{\mathrm{N}} \mathrm{N}$ threshold should then still have narrow widths.

In this paper we report on studies of antiproton nucleon annihilation by stopping antiprotons in a liquid deuterium target. Annihilation in $\mathrm{D}_{2}$ offers - in comparison to annihilation in $\mathrm{H}_{2}$ - additional possibilities which we have investigated. In particular the discussion of $\bar{p} n$ annihilation frequencies and their relation to the corresponding $\bar{p} p$ frequencies offers additional insights. We also report on a search for narrow quasinuclear states just below the $\overline{\mathrm{N}} \mathrm{N}$ threshold by measurements of the recoil momentum distribution of nucleons in $\bar{p} d$ annihilations. This method is particularly sensitive to the existence of quasinuclear bound states with binding energies of less than the pion mass which are not easily accessible using other methods.

\section{Annihilation frequencies for $\bar{p} d$ annihilation into two mesons and a nucleon}

\subsection{Data and data reduction}

The data were taken with the Crystal Barrel detector at the CERN Low Energy Antiproton Ring LEAR by stopping $200 \mathrm{MeV} / \mathrm{c}$ antiprotons in a liquid deuterium target. Details of the detector are given elsewhere [13]; here we give a short outline only of its main components. These are a barrel of $1380 \mathrm{CsI}$ crystals measuring energies and directions of $\gamma$-rays with nearly $4 \pi$ solid angle coverage, two proportional chambers and a JET drift chamber with 23 layers in which charged-particle tracks are identified and their curvatures measured in a $1.5 \mathrm{~T}$ homogeneous magnetic field. Spectator protons have mostly small momenta and do not reach the wire chambers. Therefore, annihilation on protons mostly leads to an even number of tracks, annihilation on neutrons to odd numbers.

The data presented in this paper were taken with three different types of triggers: a one-prong trigger was used in which exactly one long track was required showing a first hit in one of the 3 inner drift chamber layers and a last hit in one of the 3 outer layers. A second trigger called zero prong trigger selected events with no charged particles in the proportional chambers. We also used a minimum bias trigger to record events in which antiprotons entered the $\mathrm{D}_{2}$ target; these data were used for normalisation. Events with a pile-up flag indicating that two antiprotons entered the target within the JDC drift time were rejected for all trigger types. We define long tracks by the following requirements: a minimum of 10 hits should belong to the tracks, the first hit has to be in one of the five inner chambers, the last hit in one of the five outer layers.

We first discuss annihilation on neutrons, i.e. events in which the number of tracks is odd. We select events (from the one prong and the minimum bias data) with exactly one or exactly three long tracks with a total charge of -1 and with at most four photons. The missing mass square was calculated

$$
M M^{2}=\left(E_{\overline{\mathrm{p}} \mathrm{d}}-\sum E_{i}\right)^{2}-\left(\sum p_{i}\right)^{2}
$$

with the sum extending over all seen charged and neutral particles, and it was required that the missing particle had a mass consistent with that of a proton:

$$
\left|M M^{2}-m_{\mathrm{p}}^{2}\right|<0.5 \mathrm{GeV}^{2}
$$

Monte Carlo simulation showed that this cut does not lead to significant losses in the number of good events. But the cuts are sufficient to identify the signals and to keep the background at an acceptable level.

Fig. 1 shows the invariant mass distributions for different types of events: (a,b,c) show the $\gamma \gamma$ distribution around the $\pi^{0}, \eta$ and $\eta^{\prime}$ mass. There are two spectra identifying the reaction $\overline{\mathrm{p}} \mathrm{d} \rightarrow \pi^{-} \omega \mathrm{p}$ : Fig. (d) and (e) show the $\pi^{0} \gamma$ and $\pi^{+} \pi^{-} \pi^{0}$ invariant mass spectra for one-prong and three-prong data. The three-prong results are from minimum bias data and hence of smaller statistical accuracy. In 
(f) the $\pi^{0} \pi^{0}$ invariant mass for one-prong data is shown. A signal due to $K_{\mathrm{S}}^{0}$ mesons in their $2 \pi^{0}$ decay mode is clearly seen. Similar spectra but with reduced statistics are obtained using the minimum bias data. The $\pi^{-} \pi^{0}$ event rates in Fig. 1a and in the corresponding plot extracted from minimum bias data are used for normalisation.

For annihilations on protons we select events with 4 or $5 \gamma$ 's and no charged particles. Energy and momentum conservation is imposed by a kinematic fit and compatibility of two pairs of photons with the $\pi^{0}$ or $\eta$ mass in a kinematic fit with three constraints. Thus the threemomentum of the neutron is determined. The data show prominant signals from $\overline{\mathrm{p}} \mathrm{d} \rightarrow \pi^{0} \pi^{0} \mathrm{n}$ and $\pi^{0} \eta \mathrm{n}$. Their frequencies were determined in [14]. For events fitting the $\pi^{0} \pi^{0} \gamma+$ neutron or $\eta \pi^{0} \gamma+$ neutron hypothesis we show in Fig. 2 the $\pi^{0} \gamma$ invariant mass distribution; the $\omega$ contributions are determined from these figures. The signals are extracted from the minimum bias and from the zero prong data, those shown are the signals from the zero prong data.
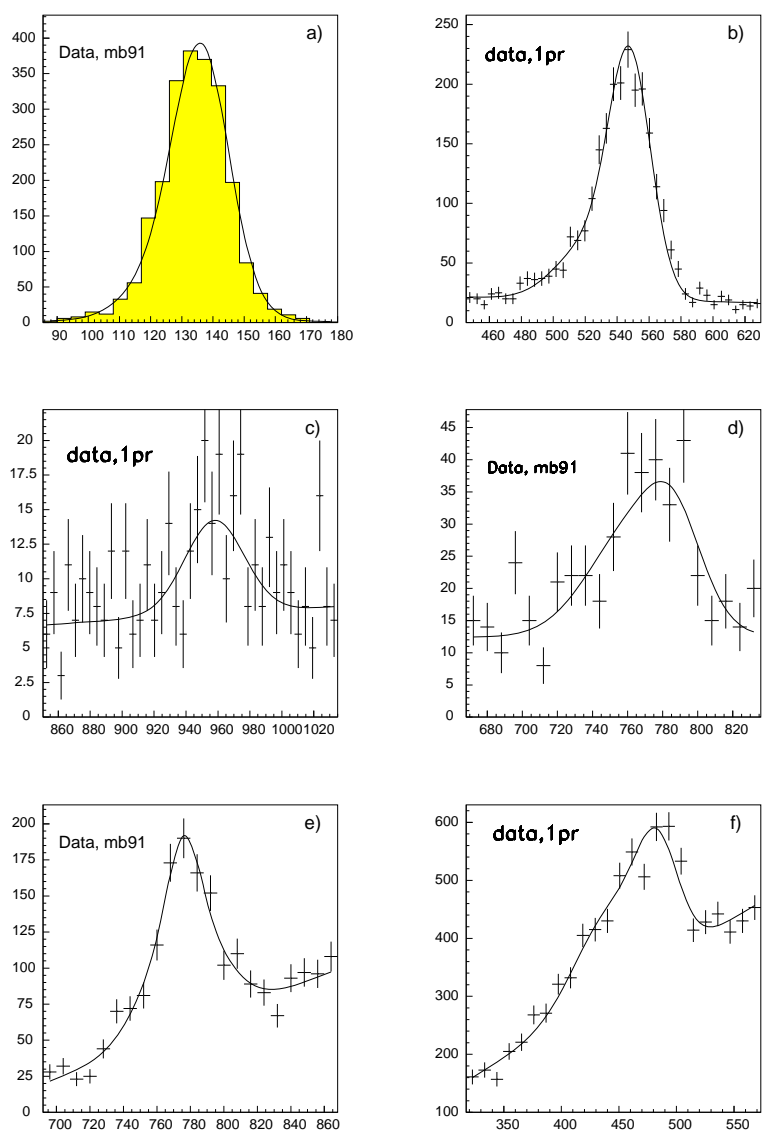

Fig. 1. Invariant mass distributions showing reactions $\bar{p} d \rightarrow$ proton $+\pi^{-} \pi^{0}, \pi^{-} \eta, \quad \pi^{-} \eta^{\prime}, \quad \pi^{-} \omega$ with $\omega \rightarrow \pi^{0} \gamma$ and $\omega \rightarrow \pi^{+} \pi^{-} \pi^{0}$ and $K^{-} K^{0}$. See text for details.
In order to determine branching ratios we simulated the corresponding reactions by Monte Carlo. The simulation requires knowledge of the proton momentum distributions which are partly not measured since high momentum protons are vetoed by the trigger. Therefore, we have used related neutron spectator distributions which can be determined from zero prong data for all neutron momenta. The proton momentum distribution in the reaction $\overline{\mathrm{p}} \rightarrow \pi^{-} \omega \mathrm{p}$ is thus assumed to be identical to the neutron momentum distribution in the reaction $\overline{\mathrm{p}} \mathrm{d} \rightarrow \pi^{0} \omega \mathrm{n}$. The latter is obtained from a kinematic fit imposing energy and momentum conservation, two $\pi^{0}$ masses and the $\omega$ mass (4 constraints). Simulated events undergo the same analysis chain as real data; they serve to define detector response functions and the detection and reconstruction efficiencies. The response functions are then used to fit the experimental data. Data and fit are shown in Fig. 1 and Fig. 2, the number of events and their reconstruction efficiencies in their respective decay modes are listed in Table 1. Contributions from competing channels to the signals in Fig. 1 are negligible.

\begin{tabular}{|ccccc|}
\hline $\begin{array}{c}\text { Reaction } \\
\overline{\mathrm{p}} \rightarrow\end{array}$ & decay & $\begin{array}{c}\text { events } \\
N_{\text {events }}\end{array}$ & $\begin{array}{c}\text { efficiency } \\
\epsilon_{r}\end{array}$ & data \\
\hline$\pi^{-} \pi^{0} \mathrm{p}$ & $\gamma \gamma$ & $2451 \pm 174$ & $0.38 \pm 0.04$ & min bias \\
$\pi^{-} \pi^{0} \mathrm{p}$ & $\gamma \gamma$ & $69657 \pm 3170$ & $0.38 \pm 0.04$ & one prong \\
$\pi^{-} \eta \mathrm{p}$ & $\gamma \gamma$ & $2529 \pm 360$ & $0.31 \pm 0.03$ & one prong \\
$\pi^{-} \eta^{\prime} \mathrm{p}$ & $\gamma \gamma$ & $103 \pm 47$ & $0.31 \pm 0.04$ & one prong \\
$\pi^{0} \omega \mathrm{n}$ & $\pi^{0} \gamma$ & $6800 \pm 300$ & $0.21 \pm 0.02$ & zero prong \\
$\eta^{0} \mathrm{n}$ & $\pi^{0} \gamma$ & $4900 \pm 200$ & $0.24 \pm 0.02$ & zero prong \\
$\pi^{-} \omega \mathrm{p}$ & $\pi^{+} \pi^{-} \pi^{0}$ & $787 \pm 100$ & $0.079 \pm 0.011$ & min bias \\
$\pi^{-} \omega \mathrm{p}$ & $\pi^{0} \gamma$ & $306 \pm 32$ & $0.34 \pm 0.02$ & min bias \\
$\mathrm{K}^{-} \mathrm{K}^{0} \mathrm{p}$ & $\pi^{0} \pi^{0}$ & $2840 \pm 237$ & $0.124 \pm 0.02$ & one prong \\
\hline
\end{tabular}

Table 1. Number of events assigned to specific reactions and their respective detection efficiencies.

\subsection{Annihilation frequencies}

Annihilation frequencies for specific reactions are derived by use of the relation

$$
B R=\frac{N_{\text {events }}}{\epsilon_{\text {reconstruction }} \epsilon_{\text {decay }} N_{\overline{\mathrm{p}} \mathrm{d}}}
$$

where the decay frequencies of the detected mesons into the observed final state $\epsilon_{\text {decay }}$ are taken from [15]. The error in $N_{\text {events }}$ is the statistical error of the fit; the error in $\epsilon_{\text {reconstruction }}$ includes the error in their construction efficiency and in the uncertainty of the momentum distribution of the surviving nucleon.

The determination of the numbers of antiproton annihilations in $\mathrm{D}_{2}$ for the three data sets is different. In 

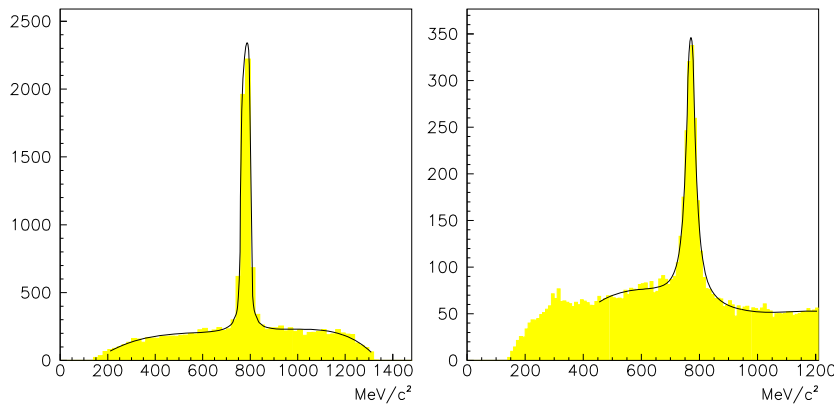

Fig. 2. Invariant $\pi^{0} \gamma$ mass distributions from $\bar{p} d \rightarrow$ neutron plus $\pi^{0} \pi^{0} \gamma(\mathrm{a})$ and $\eta \pi^{0} \gamma(\mathrm{b})$, respectively. See text for details.

case of the minimum bias data it is given by the number of events recorded on tape corrected for antiprotons stopping in the entrance window. This fraction depends on the definition of the vertex (by 2, 3 or 4 tracks) and is different for different run periods. Its values vary from $1.9 \%$ to $4.8 \%$. Since it is unknown if antiproton stopping in the entrance counter contribute to the signal, we apply a correction of $(0.98 \pm 0.02)$ to the number of recorded events. The number of antiproton stops $N_{\bar{p} d}$ in the one prong triggered data sample is determined by normalizing the number of $\pi^{-} \pi^{0} \mathrm{p}$ events to the minimum bias data; for the zero prong data $N_{\bar{p} d}$ is found by normalising the number of $\pi^{0} \pi^{0} \mathrm{n}$ events to the branching ratio for the latter reaction which has been determined before [14]. We find:

$$
\begin{aligned}
& \mathrm{N}_{\overline{\mathrm{p}} \mathrm{d}}(\mathrm{min} \text { bias })=(1.81 \pm 0.09) \cdot 10^{6} \\
& \mathrm{~N}_{\overline{\mathrm{p}} \mathrm{d}} \text { (one prong) }=(51.3 \pm 9.1) \cdot 10^{6} \\
& \mathrm{~N}_{\bar{p} \mathrm{~d}} \text { (zero prong) }=(92.4 \pm 11.9) \cdot 10^{6}
\end{aligned}
$$

From the numbers given above we derive annihilation frequencies which are listed in Table 2 and 3 . For the reaction $\overline{\mathrm{p}} \mathrm{d} \rightarrow \pi^{-} \omega \mathrm{p}$ we get 2 numbers; one for $\omega \rightarrow \pi^{0} \gamma$ and one for $\omega \rightarrow \pi^{+} \pi^{-} \pi^{0}$. The two respective values, (5.92 \pm $0.84) \cdot 10^{-3}$ and $(6.29 \pm 1.23) \cdot 10^{-3}$, are compatible and we list the mean value.

In Table 2 we compare some of the branching ratios for $\bar{p} n$ annihilations with values reported elsewhere. For this comparison we have multiplied our branching ratio by a factor of 2 to account for annihilation on protons. The agreement between the different results is fair. Note that our value was determined without cut on the proton momentum, in contrast to the other analyses. Table 3 includes frequencies which have been determined before $[14,16]$ and relevant branching ratios for $\bar{p} p$ annihilation at rest $[7,17]$ which will be used in the discussion presented in the next section.

\section{Interpretation}

\subsection{S- versus $P$-capture in $\overline{\mathbf{p}} \mathbf{d}$ annihilations}

Antiprotons stopping in liquid $\mathrm{D}_{2}$ form $\overline{\mathrm{p}} \mathrm{d}$ atoms. As in the case of $\mathrm{H}_{2}$, annihilation occurs from states with large

\begin{tabular}{|cc|c|c|}
\hline$\overline{\mathrm{p}} \rightarrow \pi^{-} \omega$ & & $\overline{\mathrm{p} n} \rightarrow \pi^{-} \pi^{0}$ & $\overline{\mathrm{p} n} \rightarrow \mathrm{K}^{0} \mathrm{~K}^{-}$ \\
\hline$[18]$ & $41 \pm 8$ & $<70$ & $14.7 \pm 2.1$ \\
{$[18]$} & $38 \pm 18$ & & \\
{$[18]$} & $180 \pm 30$ & & \\
{$[18]$} & $60 \pm 10$ & & \\
{$[18]$} & $108 \pm 29$ & & \\
{$[18]$} & $132 \pm 43$ & & \\
{$[19]$} & $85 \pm 11$ & & \\
\hline this work & $121 \pm 14$ & & \\
\hline
\end{tabular}

Table 2. Comparison of branching ratios in $\bar{p} d$ annihilation determined here with previously reported values. The ratios are multiplied by a factor 2 to account for annihilations on neutrons. All frequencies are given in units of $10^{-4}$.

principle quantum numbers $n$ and small angular momenta l. We consider $l=0$ and $l=1$ only. The total spin of the $\overline{\mathrm{p}} \mathrm{d}$ system may be $j=1 / 2$ or $j=3 / 2$. The antiproton annihilates on the proton or neutron. The annihilating $\overline{\mathrm{p}} \mathrm{N}$ system may be in a relative $S$-wave with $\mathrm{L}=0$ or in a relative $\mathrm{P}$-wave with $\mathrm{L}=1$. As was pointed out by Bizzarri et al. [20], S- and P-wave annihilation may occur from $l=0$ states of the antiprotonic deuterium atom. If $\mathrm{L}$ and $l$ are different the spectator particles carries the additional angular momentum. Often, annihilation frequencies are determined after applying a cut on the momentum of the spectator particle. The fraction of S-wave and $\mathrm{P}$-wave annihilation depends, however, on the spectator momentum and hence on the spectator momentum cut.

Conflicting results have been obtained on the fraction of P-wave annihilation for antiprotons stopping in liquid deuterium $P_{L D}$, possibly due to the use of different spectator momentum cuts. In [21] it was suggested that the fraction of $\mathrm{P}$-wave annihilation in liquid deuterium can be determined from the frequencies of $\bar{p} d$ annihilations into two pions. The $\mathrm{P}$-wave fraction was determined from $\overline{\mathrm{p}} \mathrm{N}$ annihilations into two mesons and using charge independence of strong interactions. The frequency of the reaction $\overline{\mathrm{p}} \mathrm{d} \rightarrow \pi^{+} \pi^{-} \mathrm{n}, f_{L D_{2}}\left(\pi^{+} \pi^{-} \mathrm{n}\right)$, may be split into an $\mathrm{S}$-wave contribution and a $\mathrm{P}$-wave contribution. The reaction $\overline{\mathrm{p}} \mathrm{d} \rightarrow \pi^{-} \pi^{0} \mathrm{p}$ is allowed only from the $\mathrm{S}$-wave in the antiproton-neutron subsystem. Isospin invariance requires

$$
f_{L D_{2}, S-\text { wave }}\left(\pi^{+} \pi^{-} \mathrm{n}\right)=\frac{1}{2} \cdot f_{L D_{2}}\left(\pi^{-} \pi^{0} \mathrm{p}\right)
$$

$\overline{\mathrm{p}} \mathrm{d} \rightarrow \pi^{0} \pi^{0} \mathrm{n}$ is only allowed from P-wave in $\overline{\mathrm{p}} \mathrm{p}$. Isospin invariance now predicts that

$$
f_{L D_{2}, P-\text { wave }}\left(\pi^{+} \pi^{-} \mathrm{n}\right)=2 \cdot f_{L D_{2}}\left(\pi^{0} \pi^{0} \mathrm{n}\right)
$$

The total $\pi^{+} \pi^{-}$frequency in deuterium is given by the sum of $\mathrm{S}$-wave and $\mathrm{P}$-wave annihilation:

$$
f_{L D_{2}}\left(\pi^{+} \pi^{-} \mathrm{n}\right)=\frac{1}{2} \cdot f_{L D_{2}}\left(\pi^{-} \pi^{0} \mathrm{p}\right)+2 \cdot f_{L D_{2}}\left(\pi^{0} \pi^{0} \mathrm{n}\right)(6)
$$

The left hand side was determined to be $(42 \pm 12) \cdot 10^{-4}$ in [21]; we find $(29.9 \pm 2.5) \cdot 10^{-4}$ for the right hand side. 


\begin{tabular}{|c|c|c|c|c|c|c|c|c|c|}
\hline $\begin{array}{c}\overline{\mathrm{p}} \mathrm{N} \text { initial state } \\
{ }^{2 s+1} L_{J}\end{array}$ & $\begin{array}{c}\text { Reaction } \\
\overline{\mathrm{p} p} \rightarrow\end{array}$ & frequency & Ref. & $\begin{array}{c}\text { Reaction } \\
\overline{\mathrm{p}} \mathrm{d} \rightarrow\end{array}$ & frequency & Ref. & $\begin{array}{c}\text { Reaction } \\
\overline{\mathrm{p}} \mathrm{d} \rightarrow\end{array}$ & frequency & Ref. \\
\hline${ }^{3} \mathrm{~S}_{1}$ & $\pi^{+} \pi^{-}$ & $16.8 \pm 1.3$ & {$[17]$} & $\pi^{+} \pi^{-} \mathrm{n}$ & $42 \pm 12$ & {$[21]$} & $\pi^{-} \pi^{0} \mathrm{p}$ & $36.1 \pm 4.9$ & \\
\hline${ }^{3} \mathrm{P}_{0}$ & $\pi^{0} \pi^{0}$ & $6.93 \pm 0.43$ & {$[17]$} & $\pi^{0} \pi^{0} \mathrm{n}$ & $5.90 \pm 0.30$ & {$[14]$} & - & & \\
\hline${ }^{3} \mathrm{P}_{0}$ & $\pi^{0} \eta$ & $2.12 \pm 0.12$ & {$[17]$} & $\pi^{0} \eta \mathrm{n}$ & $2.46 \pm 0.12$ & {$[14]$} & $\pi^{-} \eta p$ & $4.06 \pm 1.00$ & \\
\hline${ }^{3} \mathrm{P}_{0}$ & $\pi^{0} \eta^{\prime}$ & $1.23 \pm 0.13$ & {$[17]$} & - & & & $\pi^{-} \eta^{\prime} \mathrm{p}$ & $2.98 \pm 1.52$ & \\
\hline${ }^{1} \mathrm{~S}_{0}$ & $\rho^{ \pm} \pi^{\mp}$ & $9.2 \pm 4.0$ & {$[11]$} & - & & & $\rho^{-} \pi^{0} \mathrm{p}$ & $6.9 \pm 1.5$ & [16] \\
\hline${ }^{3} \mathrm{~S}_{1}$ & $\pi^{0} \omega$ & $57.3 \pm 4.7$ & {$[17]$} & $\pi^{0} \omega \mathrm{n}$ & $41.8 \pm 3.9$ & & $\pi^{-} \omega \mathrm{p}$ & $60.4 \pm 6.9$ & \\
\hline${ }^{3} S_{1}$ & $\eta \omega$ & $151 \pm 12$ & {$[17]$} & $\eta \omega \mathrm{n}$ & $67.1 \pm 8.1$ & & - & & \\
\hline${ }^{2 s+1} L_{J}$ & $\overline{\mathrm{p} p} \rightarrow$ & & & $\overline{\mathrm{p} p} \rightarrow$ & & & $\overline{\mathrm{p}} \mathrm{d} \rightarrow$ & & \\
\hline${ }^{3} \mathrm{~S}_{1}$ & $\mathrm{~K}^{+} \mathrm{K}^{-}$ & $9.1 \pm 0.5$ & {$[17]$} & $\mathrm{K}_{\mathrm{S}}^{0} \mathrm{~K}_{1}^{0}$ & $8.5 \pm 0.7$ & [32]. & $\mathrm{K}^{0} \mathrm{~K}^{-} \mathrm{p}$ & $14.2 \pm 3.6$ & \\
\hline
\end{tabular}

Table 3. Isospin relations in $\overline{\mathrm{p}} \mathrm{N}$ annihilation. The dominant initial state is given as ${ }^{2 s+1} L_{J}$; contributions from other channels are at the $10 \%$ level or forbidden. All frequencies are given in units of $10^{-4}$. If no reference is given, the branching ratio is determined in this work. Derived values for S-state only are given for the reactions $\overline{p p} \rightarrow \pi^{+} \pi^{-}$and $\overline{p p} \rightarrow \mathrm{K}^{+} \mathrm{K}^{-}$; the $\mathrm{P}$-state contribution is subtracted (see [17]).

In previous experiments, the $\mathrm{P}$-wave contribution was determined using (6) from the ratio

$$
r=f_{L D_{2}}\left(\pi^{-} \pi^{0} \mathrm{p}\right) / f_{L D_{2}}\left(\pi^{+} \pi^{-} \mathrm{n}\right)
$$

which was measured to be $0.68 \pm 0.07$ [21], $0.70 \pm 0.05[22]$, $0.55 \pm 0.05[22]$ and $2.07 \pm 0.05$ [23]. In this experiment, the $\mathrm{S}$-wave and P-wave part are measured directly. We obtain

$$
\begin{aligned}
\frac{P_{L D_{2}}}{S_{L D_{2}}+P_{L D_{2}}} & =\frac{2 \cdot f_{L D_{2}}\left(\pi^{0} \pi^{0} \mathrm{n}\right)}{\frac{1}{2} \cdot f_{L D_{2}}\left(\pi^{-} \pi^{0} \mathrm{p}\right)+2 \cdot f_{L D_{2}}\left(\pi^{0} \pi^{0} \mathrm{n}\right)} \\
& =0.40 \pm 0.04
\end{aligned}
$$

and $r=0.86 \pm 0.27$

However it has been pointed out [24], that $r$ and equ. (8) determine the fraction of the reaction $\overline{\mathrm{p}} \mathrm{d} \rightarrow \pi^{+} \pi^{-} \mathrm{n}$ which proceeds from P-states; not the fraction of P-state annihilation in $\bar{p} d$ interactions at rest.

An absolute estimate of the $\mathrm{P}$-wave annihilation fraction can be derived by comparing the frequencies for annihilation into $\pi^{0} \pi^{0}$ in liquid $\mathrm{H}_{2}$ and liquid $\mathrm{D}_{2}$. If the $\mathrm{P}-$ wave fractions were the same, the frequency in $\mathrm{D}_{2}$ should be $\frac{1}{2} \cdot 6.93 \cdot 10^{-4}$. This is not the case, the $\pi^{0} \pi^{0}$ frequency exceeds this value by the ratio $2 \cdot 5.90 / 6.93$. In $\mathrm{H}_{2}$ the $\mathrm{P}-$ wave fraction is $(13 \pm 4) \%$ [25]. Hence we expect a P-wave fraction of $(22 \pm 4) \%$. The same argument can be used for the $\pi \eta$ and $\pi \eta^{\prime}$ final states from which we derive a P-wave fraction of $(30 \pm 10) \%$.

In any case the P-state fraction $f_{\mathrm{P}}($ liq $)$ does not correspond to the fraction of antiproton deuteron annihilations in which the antiproton has unit orbital angular momentum $l=1$ with respect to the deuteron. Instead, it refers to the angular momentum state of the annihilating $\overline{\mathrm{p}} \mathrm{N}$ system. The reaction $\overline{\mathrm{p}} \mathrm{d} \rightarrow \Delta^{0}(1232) \pi^{0}$ is e.g. allowed from S-wave orbitals of the $\bar{p} d$ atom (with $l=0$ ), and the $\Delta^{0}$ may nevertheless decay into $\pi^{0} \mathrm{n}$. The $2 \pi^{0}$ thus observed in the final state reflect $\mathrm{L}=1$ between antiproton and proton at the time when annihilation occurred. The frequency for $\overline{\mathrm{p}} \mathrm{d} \rightarrow \pi^{0} \pi^{0} \mathrm{n}$ (which identifies P-states in the $\bar{p}$ p subsystem) thus depends not only on the fraction of antiprotons annihilating from P-wave orbitals of the $\bar{p} d$ atom but also on the probability of formation of nucleon resonances and of rescattering pions from annihilation off the surviving nucleon. A cascade calculation has shown [26] that the contributions of $\mathrm{S}$ and $\mathrm{P}$ angular momentum states of the proton with respect to the proton or to the deuteron, respectively, in $\bar{p} p$ and $\bar{p} d$ atoms are the same. An increase in P-state capture probability in $\mathrm{D}_{2}$ can therefore be expected due to the additional chance of antiprotons stopped in $\mathrm{D}_{2}$ to be captured from $\mathrm{S}$-wave orbitals of the atom but in a $\mathrm{P}$-wave angular momentum state between the antiproton and annihilating nucleon. These arguments were previously discussed by Bizzarri and collaborators [20] but with fewer and less precise experimental results. A detailed discussion on S-state and P-state capture in $\bar{p} d$ annihilation can be found in [27].

\subsection{The isospin structure of the protonium wave function}

Now we turn to a discussion of initial state interactions. The wave function of antiprotonic hydrogen atoms is predicted to have an interesting isospin structure: at large distances the protonium wave function is entirely given by the Coulomb field between proton and antiproton which preserve their identity. At smaller distances pion exchange starts to play a significant role, and the protonium wave function may acquire some $\bar{n} n$ component. Hence initial state interactions may change the $\bar{p} p$ wave function into a linear superposition of $\bar{p} p$ and $\bar{n} n$ contributions. This effect had been proposed by Kaufmann and Pilkuhn [28]; a detailed study can be found in an article by Carbonell, Ihle and Richard [6].

Initial state interactions thus lead to a mixture of isospin $I=0$ and $I=1$ components of the protonium wave function with a priori unknown complex strengths $a(r)$ 


\begin{tabular}{|c|ccc|cc|}
\hline Initial state & \multicolumn{3}{|c|}{ Theory [6] } & from ref. [7] & this work \\
\hline${ }^{1} \mathrm{~S}_{0}$ & 0.68 & 0.68 & 0.8 & $0.72_{-0.18}^{+0.24}$ & $0.50_{-0.29}^{+0.48}$ \\
${ }^{3} \mathrm{~S}_{1}$ & 1.22 & 0.95 & 1.26 & $1.17_{-0.28}^{+0.39}$ & $1.17_{-0.23}^{+0.30}$ \\
${ }^{3} \mathrm{P}_{0}$ & 0.03 & 0.03 & 0.053 & $1.16 \pm 0.34$ & $0.41_{-0.09}^{+0.11}$ \\
\hline
\end{tabular}

Table 4. Ratio of isovector to isoscalar fraction of the protonium wave function for various initial states ${ }^{2 s+1} L_{J}$. The 3 theoretical values correspond to different $\overline{\mathrm{N}} \mathrm{N}$ potentials.

and $b(r)$ :

$$
\mid \overline{\mathrm{p}} \mathrm{p}(r)>=\frac{1}{\sqrt{2}} \cdot\left(a(r)\left|I=0, I_{3}=0>+b(r)\right| I=1 ; I_{3}=0>\right)
$$

In the absence of initial state interactions $a(r)=b(r)=1$, independent of the $\overline{\mathrm{N}} \mathrm{N}$ separation. In general, we have $|a(r)|^{2}+|b(r)|^{2}=2$. Within a potential model of $\overline{\mathrm{N}} \mathrm{N}$ interactions with a phenomenological annihilation potential we may define global isospin components by integrating $a(r)$ and $b(r)$ over the $\bar{N} \mathrm{~N}$ density folded with the annihilation potential.

$$
a=\int a^{*}(r) \psi^{*}(r) V_{a n n}(r) a(r) \psi(r) d^{3} r
$$

The global isovector fraction is defined in an equivalent way. Due to annihilation, the isospin structure constants $a(r)$ and $b(r)$ can be complex; they depend on the quantum numbers of the initial states from which annihilation occurs. In Table 4 we give a list of values for $|b|^{2} /|a|^{2}=\gamma$ for various initial states calculated using different $\overline{\mathrm{NN}}$ potentials. We also quote the results of a first attempt to determine the global strength of initial state interactions from data on $\bar{p}$ p annihilation into two mesons, and give the results obtained here.

The values are derived as follows. The branching ratio $\overline{\mathrm{p}} \mathrm{d} \rightarrow \pi^{-} \omega$ defines the strength of the transition operator

$$
B R\left(\overline{\mathrm{p}} \mathrm{d} \rightarrow \pi^{-} \omega \mathrm{p}\right)=\frac{1}{2} T_{\pi \omega}^{2}
$$

The factor $1 / 2$ accounts for the Clebsch Gordon coefficient for $\bar{p}$ annihilating on the neutron. In annihilations on the proton, the expected rate is reduced since the $\bar{p} p$ wave function comprises an isospin-zero part. Hence

$$
B R\left(\overline{\mathrm{p}} \mathrm{d} \rightarrow \pi^{0} \omega \mathrm{n}\right)=\frac{1}{4} b^{2} T_{\pi \omega}^{2}
$$

Neglecting losses due to $\pi^{0}$ or $\omega$ inelastic scattering off the neutron we can relate the branching ratio in (12) with

$$
B R\left(\overline{\mathrm{p} p} \rightarrow \pi^{0} \omega\right)=\frac{1}{2} b^{2} T_{\pi \omega}^{2}
$$

Thus we obtain, using the branching ratios of Table 3 , $b^{2}=1.38 \pm 0.20($ from $(12 / 11))$ and $b^{2}=0.95 \pm 0.13$ (from (13/11)) giving a weighted mean of $b^{2}=1.08 \pm 0.11$. The corresponding number for ${ }^{1} \mathrm{~S}_{0}$ is derived from the $\rho \pi$ frequencies. The branching ratio for $\bar{p} p \rightarrow \rho^{+} \pi^{-}$is smaller than that for $\overline{\mathrm{p}} \rightarrow \rho^{-} \pi^{0} \mathrm{p}$ resulting in $b^{2}=0.67 \pm 0.32$.

We notice (see Table 4) good agreement between the theoretical prediction and the two experimental determinations using different branching ratios (in [7] no deuterium data were used). However, in both cases, predicted and measured isoscalar and isovector parts of the $\bar{p} p$ wave function in the ${ }^{3} \mathrm{~S}_{1}$ state have about the same size; thus we can conclude that initial state interactions are not needed but are also not excluded by data. The effect of initial state interactions in both levels is too small to allow discriminating conclusions.

The effect of initial state interactions is much more pronounced in atomic P-states; hence the effect can be studied much better. First we notice that $\overline{\mathrm{p}} \mathrm{N}$ annihilation into $\pi \eta$ or $\pi \eta^{\prime}$ proceeds dominantly via the ${ }^{3} \mathrm{P}_{0}$ state and not via ${ }^{3} \mathrm{P}_{2}$. The preference for ${ }^{3} \mathrm{P}_{0}$ is due to the larger hadronic width of this state [6] and leads to an enhancement of the $\mathrm{P}$ capture rate for $\pi^{0} \pi^{0}, \pi^{0} \eta$ or other rates for annihilation into 2 neutral pseudoscalar mesons. On the other hand, the predicted $(b / a)^{2}$ is between 0.03 and 0.053 [6] implying that $\mathrm{BR}_{\bar{p} p \rightarrow \pi^{0} \eta}$ and $\mathrm{BR}_{\bar{p} p \rightarrow \pi^{0} \eta^{\prime}}$ - which proceed via the (supposedly small) isovector component of the protonium wave function - should be much smaller than $\mathrm{BR}_{\bar{p} d \rightarrow \pi^{-} \eta p}$, respectively. This is evidently not the case. Replacing the $\omega$ by an $\eta$ in (11-13) we find $b^{2}=1.21 \pm 0.3$ (from $(12 / 11)$ ) and $b^{2}=0.52 \pm 0.13$ (from $(13 / 11)$ ). From $\pi \eta^{\prime}$ production we deduce $b^{2}=0.41 \pm 0.22$. The results are not consistent but certainly not very small. Scaling the errors to enforce compatibility results in a weighted mean of $b^{2}=0.58 \pm 0.17$ in disagreement with values obtained from $\overline{\mathrm{N}} \mathrm{N}$ potential models but still in $2.5 \sigma$ agreement with the assumption of no initial state interactions.

We have neglected the role which the fraction of S-state and P-state capture may have on the determination of $b^{2}$. This is likely justified in case of $\pi \omega$ or $\pi \rho$ production since these decays are allowed from $\mathrm{S}$ - and P-states. But this must be wrong in the case of $\pi \eta$ or $\pi \eta^{\prime}$ production which is forbidden in S-state annihilation. The P-state fraction does not influence the ratio $(12 / 11)$ where we compare two reactions in $\mathrm{D}_{2}$. But it does change the ratio $(13 / 11)$ since P-state capture is larger in $\mathrm{D}_{2}$ than in $\mathrm{H}_{2}$. Using the results from section 3.1 we estimate $\mathrm{b}^{2} \sim 1$. We conclude that there is no experimental evidence for a large role of initial state interactions for $\overline{\mathrm{N}} \mathrm{N}$ annihilation as predicted from $\overline{\mathrm{N}} \mathrm{N}$ potential models.

We recall here that $\rho-\omega$ interference observed in $\overline{\mathrm{p}} \rightarrow \pi^{+} \pi^{-} \eta[29]$ and $\overline{\mathrm{p}} \rightarrow \pi^{+} \pi^{-} \pi^{0}$ [30] also provides evidence for the assumption that the initial state in $\overline{\mathrm{N}} \mathrm{N}$ annihilation is not affected by meson exchange processes. In Fig. 3 the respective $\pi^{+} \pi^{-}$mass distributions are compared to the distribution obtained from $e^{+} e^{-} \rightarrow \pi^{+} \pi^{-}$annihilation. Interference of $\rho$ and $\omega$ is clearly observed in all three reactions even though the shapes look different. This is due to the different $\omega: \rho$ production ratios. These are $\sim 1 / 9$ for $e^{+} e^{-}$from vector meson dominance, $\sim 1 / 3$ for $\overline{\mathrm{p}} \mathrm{p} \rightarrow \pi^{0}(\omega / \rho)$ and $\sim 4 / 1$ for $\overline{\mathrm{p}} \mathrm{p} \rightarrow \eta(\omega / \rho)$. Surprisingly, the phase of the interference is nearly identical. In $e^{+} e^{-}$annihilation the phase is given by vector meson dominance and 

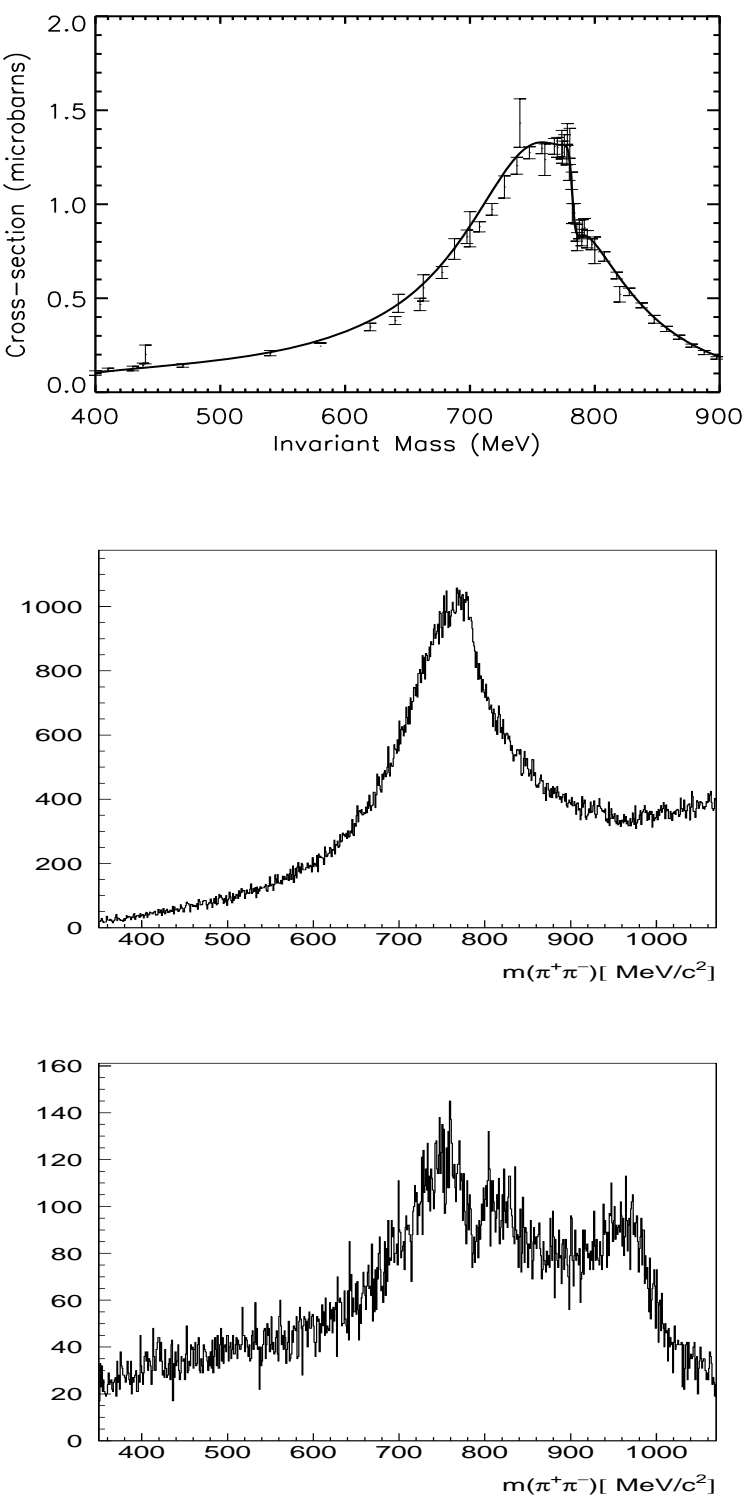

Fig. 3. Invariant mass distributions of $\pi^{+} \pi^{-}$from $e^{+} e^{-}$ annihilation (top), from $\bar{p} p \rightarrow \pi^{+} \pi^{-} \pi^{0}$ (middle) and from $\overline{\mathrm{p}} \mathrm{p} \rightarrow \pi^{+} \pi^{-} \eta$ (bottom).

is nearly real [31]. In $\bar{p} p$ annihilation there seems to be no additional phase between the isovector and isoscalar components. $\overline{\mathrm{N} N}$ potential models suggest an additional phase because of differences in the $(\mathrm{I}=0)$ and $(\mathrm{I}=1)$ annihilation potentials [6].

\subsection{A dynamical selection rule in $\bar{N} N$ annihilation into $\mathrm{K} \overline{\mathrm{K}}$}

In Table 5 we collect the branching ratios for $\overline{\mathrm{N}} \mathrm{N} \rightarrow \mathrm{K} \overline{\mathrm{K}}$. Assuming that the $\bar{p} p$ wave function is given by (9) with

$$
\begin{array}{lc}
\overline{\mathrm{p}} \rightarrow \mathrm{K}^{+} \mathrm{K}^{-} & \frac{1}{2}\left(\left|A_{0}\right|^{2}+\left|A_{1}\right|^{2}\right)+\operatorname{Re}\left(A_{0} A_{1}^{*}\right) \\
\overline{\mathrm{p}} \rightarrow \mathrm{K}_{\mathrm{S}}^{0} \mathrm{~K}_{1}^{0} & \frac{1}{2}\left(\left|A_{0}\right|^{2}+\left|A_{1}\right|^{2}\right)-\operatorname{Re}\left(A_{0} A_{1}^{*}\right) \\
\overline{\mathrm{p} d} \rightarrow \mathrm{K}^{0} \mathrm{~K}^{-} \mathrm{p} & \left|A_{1}\right|^{2}
\end{array}
$$

$\mathrm{a}=\mathrm{b}=1$, then $A_{0}, A_{1}$ are the isoscalar and isovector amplitudes for $\bar{p} p$ annihilation into $\bar{K} K$.

The data are compatible with $A_{0}=0$, hence with the assumption that the isoscalar part of the $\bar{p} p$ wave function does not couple to the $\mathrm{K} \overline{\mathrm{K}}$ final state. This is a striking dynamical selection rule which so far has found no explanation in $\overline{\mathrm{N} N}$ potential models. Within $\mathrm{SU}(3)$ it can be related to a general dynamical selection rule observed in $\overline{\mathrm{p}} \mathrm{p}$ annihilation at rest into $\mathrm{K} \overline{\mathrm{K}}$ or $\mathrm{K} \overline{\mathrm{K}^{*}}+$ charge conjugate [33].

\begin{tabular}{|cc|c|}
\hline${ }^{3} \mathrm{~S}_{1}$ & $\overline{\mathrm{p}} \mathrm{p} \rightarrow \mathrm{K}^{+} \mathrm{K}^{-}$ & $9.1 \pm 0.5$ \\
${ }^{3} \mathrm{~S}_{1}$ & $\overline{\mathrm{p}} \mathrm{p} \rightarrow \mathrm{K}_{\mathrm{s}}^{0} \mathrm{~K}_{1}^{0}$ & $8.5 \pm 0.7$ \\
${ }^{3} \mathrm{~S}_{1}$ & $\overline{\mathrm{p}} \mathrm{d} \rightarrow \mathrm{K}^{0} \mathrm{~K}^{-} \mathrm{p}$ & $14.2 \pm 3.6$ \\
\hline
\end{tabular}

Table 5. Branching ratios for $\bar{p} \mathrm{~N}$ annihilation into $\mathrm{K} \overline{\mathrm{K}}$. The frequencies are given in units of $10^{-4}$.

\section{Search for quasinuclear bound states}

One of the most spectacular results of $\overline{\mathrm{N}} \mathrm{N}$ potential models is the prediction of - possibly narrow - quasinuclear bound states [8,9]. The initial experimental evidence [34] faded away when LEAR started to allow high-precision experiments with antiprotons at rest [10] or in flight [35, 36]. The interest was renewed when the Asterix collaboration reported a new isoscalar tensor state, the AX(1565), which coupled strongly to $\overline{\mathrm{N}} \mathrm{N}$, which was not seen before in non- $\overline{\mathrm{N}} \mathrm{N}$ experiments and the mass of which agreed nicely with $\bar{N} N$ model predictions. The state was identified with a tensor state seen in $\overline{\mathrm{p}} \mathrm{n} \rightarrow 3 \pi^{-} 2 \pi^{+}$by Kalogeropoulos and collaborators (but at a mass of $1490 \mathrm{MeV}$ ) [19] and interpreted as quasinuclear bound state [12]. If this meson is indeed a quasinuclear bound state, $\bar{N} N$ potential models predict a series of further states. These are a scalar and a vector meson, lower in mass than the AX(1565), and a number of states clustering at about twice the proton mass. The two low-mass quasinuclear states should be much broader than the AX(1565) and may thus be undetectable. The $\overline{\mathrm{N}} \mathrm{N}$ states near to the $\overline{\mathrm{N}} \mathrm{N}$ threshold however are loosely bound and should thus be narrow. On the other hand, states close to the $\overline{\mathrm{N}} \mathrm{N}$ threshold cannot be produced in annihilation if their mass $M_{X}$ is larger than $2 M_{p}-m_{\pi}$. Such states can however be produced in reactions like

$$
\overline{\mathrm{p}} \mathrm{d} \rightarrow X+N
$$

where $\mathrm{X}$ is a bound state and $\mathrm{N}$ represents the recoiling nucleon. The momentum spectrum of spectator nucleons 


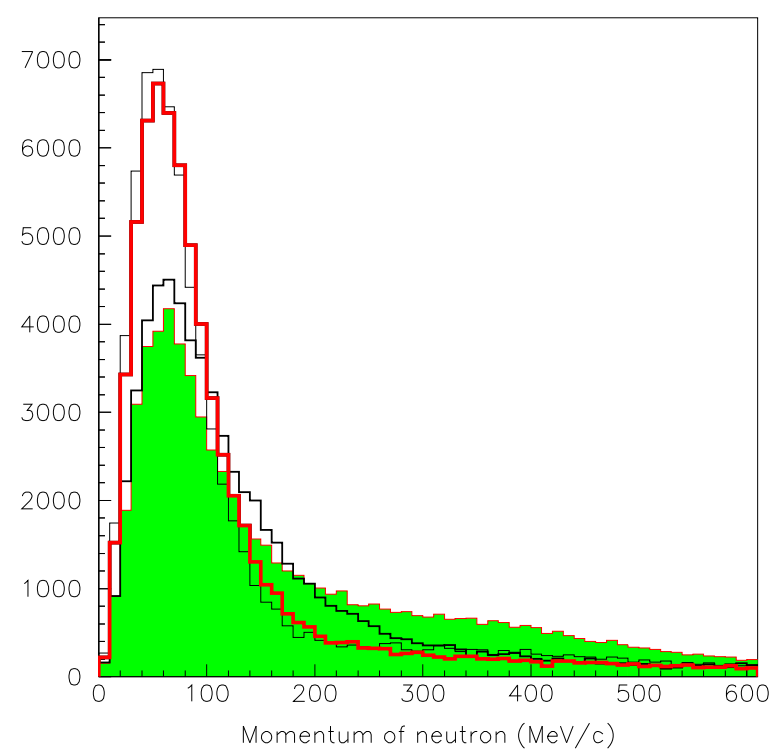

Fig. 4. Momentum distribution of the spectator neutron for $\overline{\mathrm{p}} \mathrm{d} \rightarrow k \pi^{0}$ neutron, for $\mathrm{k}=2$ (medium line thickness), $\mathrm{k}=3$ (thick), $\mathrm{k}=4$ (grey shaded), $\mathrm{k}=5$ (thin).

shows a characteristic shape in these reactions which reflects the Fermi motion of nucleons in deuterons. However, high momenta also occur due to final-state interactions or due to the production of resonances. A quasinuclear state should manifest itself as a peak in the momentum distribution if the annihilation process proceeds via the formation of an intermediate $\overline{\mathrm{N}} \mathrm{N}$ state recoiling against the residual nucleon.

We have searched for quasinuclear bound states close to the N $\overline{\mathrm{N}}$ threshold for momenta of the spectator neutron of $<1000 \mathrm{MeV} / \mathrm{c}$. In case of resonances close to this threshold, the peak falls into the region of the Fermi momentum and a deviation from the expected distribution should give evidence for the new state. Such states very close to the $\overline{\mathrm{N}} \mathrm{N}$ threshold have been claimed by different authors [37, 22, 38, 39].

The zero prong data sample were used for this search. Events with 4, 6, 8 and $10 \gamma^{\prime}$ 's underwent a kinematical fit to the $(\mathrm{k} \gamma) \mathrm{n}$ hypothesis imposing energy and momentum conservation. Fitting the neutron momentum as an unmeasured quantity, energy and momentum conservation provides one constraint (1C). 433200 events passed the fit with $1 \%$ confidence level. These data were subject to further kinematical fits using the masses of the $\pi^{0}$ and $\eta$ as additional constraints. Events are selected with a confidence level $>10 \%$, respectively. Events having a large probability to belong to background channels are rejected. Table 6 lists the number of events for the reactions considered, their reconstruction efficiencies and the branching ratios. The reconstruction efficiency $\epsilon_{\mathrm{r}}$ depends on our

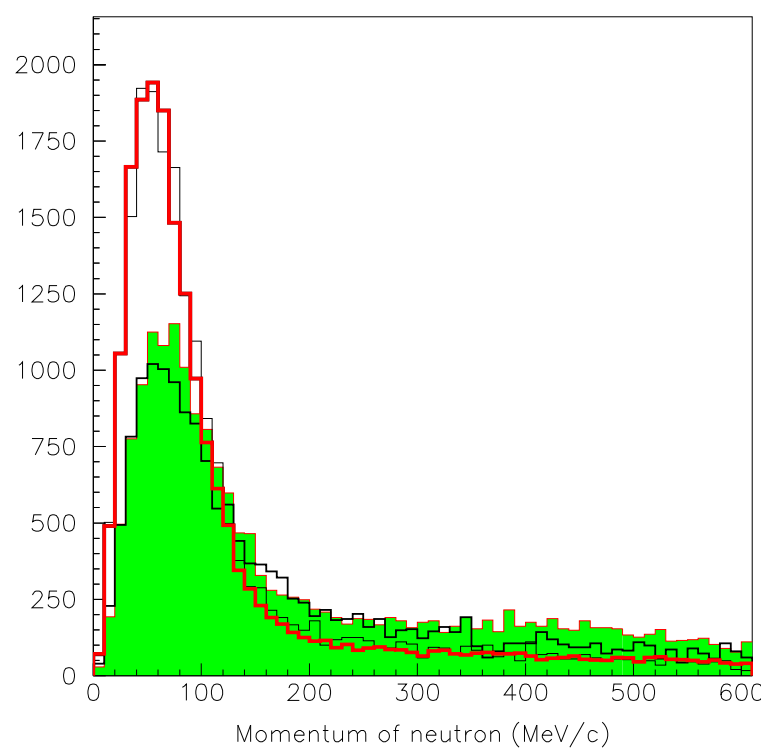

Fig. 5. Momentum distribution of the spectator neutron for $\overline{\mathrm{p}} \mathrm{d} \rightarrow \eta k \pi^{0}$ neutron,

for $\mathrm{k}=1$ (medium line thickness), $\mathrm{k}=2$ (thick), $k=3$ (grey shaded), $k=4$ (thin).

knowledge of the true neutron recoil momentum distribution. This uncertainty dominates the error in $\epsilon_{\mathrm{r}}$ which we estimate to be $10 \%$ of its magnitude. We compare the annihilation frequencies of Table 6 for $\bar{p} p$ annihilation in deuterium with those obtained in hydrogen. We expect the frequencies in $\bar{p} d$ to be smaller by a factor 2 due to the additional possibility of $\overline{\mathrm{p}} \mathrm{n}$ annihilations. In some cases (in particular in $\overline{\mathrm{p}} \mathrm{d} \rightarrow \pi^{0} \pi^{0} \mathrm{n}$ and $\overline{\mathrm{p}} \mathrm{d} \rightarrow \pi^{0} \eta \mathrm{n}$ ) the frequency in deuterium is much larger. This is due to a large probability to form nucleon resonances [14].

Figures 4 and 5 show the momentum distributions of the recoiling neutron for the different reactions. The distributions are normalised to 70000 for the $\mathrm{k} \pi^{0}$ and to 20000 for the $\eta\left(\mathrm{k} \pi^{0}\right)$ events to facilitate the comparison. A quasinuclear bound state will have a defined G-parity which can be determined from the number of pions into which the resonance decays. Hence we expect systematic differences between even and odd number of pions.

The dominant resonance $\mathrm{N} \overline{\mathrm{N}}(1870)$ as predicted on the basis of the ASTERIX data [38] should manifest itself by showing a signature in decay channels with an odd number of pions whereas decay channels with an even number of pions should have no signature from that state. Figure 4 obviously does not give evidence for such a resonance, at least not at the level observed in the ASTERIX experiment. It should be noted that the claim for the $\mathrm{N} \overline{\mathrm{N}}(1870)$ was based on a comparison of the measured proton momentum distribution with the calculated distribution. The measured distribution was broader. Probably, the differ- 


\begin{tabular}{|cccccc|cc|}
\hline$\overline{\mathrm{p}} \rightarrow$ & final state & Constraints & $\mathrm{N}_{\text {events }}$ & $\epsilon \mathrm{r}$ & $\mathrm{BR}$ & $\overline{\mathrm{p}} \mathrm{p} \rightarrow$ final state & BR \\
& $2 \pi^{0}+\mathrm{n}$ & $(3 \mathrm{C})$ & $36801 \pm 192$ & $0.459 \pm 0.047$ & $6.7 \pm 0.8$ & $2 \pi^{0}$ & $6.9 \pm 0.4[17]$ \\
$\eta \pi^{0}+\mathrm{n}$ & $(3 \mathrm{C})$ & $5904 \pm 77$ & $0.423 \pm 0.044$ & $3.1 \pm 0.4$ & $\eta \pi^{0}$ & $2.1 \pm 0.1[17]$ \\
$3 \pi^{0}+\mathrm{n}$ & $(4 \mathrm{C})$ & $69752 \pm 264$ & $0.292 \pm 0.030$ & $39.4 \pm 4.8$ & $3 \pi^{0}$ & $62.0 \pm 10.0[41]$ \\
$\eta 2 \pi^{0}+\mathrm{n}$ & $(4 \mathrm{C})$ & $19563 \pm 140$ & $0.290 \pm 0.030$ & $28.9 \pm 3.6$ & $\eta 2 \pi^{0}$ & $67.0 \pm 12.0[41]$ \\
$4 \pi^{0}+\mathrm{n}$ & $(5 \mathrm{C})$ & $49778 \pm 223$ & $0.124 \pm 0.013$ & $26.0 \pm 2.9$ & $4 \pi^{0}$ & $44.0 \pm 3.0[42]$ \\
$\eta 3 \pi^{0}+\mathrm{n}$ & $(5 \mathrm{C})$ & $7621 \pm 87$ & $0.157 \pm 0.017$ & $11.1 \pm 1.4$ & $\eta 3 \pi^{0}$ & - \\
$5 \pi^{0}+\mathrm{n}$ & $(6 \mathrm{C})$ & $28674 \pm 169$ & $0.076 \pm 0.008$ & $34.1 \pm 3.7$ & $5 \pi^{0}$ & $92.0 \pm 13.0[43]$ \\
$\eta 4 \pi^{0}+\mathrm{n}$ & $(6 \mathrm{C})$ & $5648 \pm 75$ & $0.090 \pm 0.009$ & $17.8 \pm 2.2$ & $\eta 4 \pi^{0}$ & $69.0 \pm 11.0[42]$ \\
\hline
\end{tabular}

Table 6. All frequencies are given in units of $10^{-4}$.

ences were due to the neglect of the experimental resolution in [38].

We notice that the $4 \pi$ and (to a lesser extent) the $2 \pi$ momentum distributions have higher intensities at large momenta than the $3 \pi$ and $5 \pi$ have. We believe that this effect reflects different probabilities for S-state and P-state capture. Annihilation into $2 \pi^{0}$ is strictly forbidden from $\overline{\mathrm{p} p}$ in S-states, annihilation into $4 \pi^{0}$ is not absolutely forbidden from $\mathrm{S}$-states but requires a rather complex cascade (like $\overline{\mathrm{p}} \mathrm{\rightarrow} \pi^{0} a_{2}(1650), a_{2}(1650) \rightarrow \pi^{0} f_{2}(1270)$, $\left.f_{2}(1270) \rightarrow 2 \pi^{0}\right)$. P-state annihilation of the $\bar{p} p$ system is allowed from $\mathrm{S}$-wave orbits of the $\bar{p} d$ atom; the neutron and the annihilating $\bar{p} p$ system have then one unit of angular momentum too, which implies a preference for large momenta of the surviving neutron. This conjecture is confirmed by the momentum distributions with $\eta$ mesons. Again, the distribution with large intensity at high neutron momenta, the distributions from $\overline{\mathrm{p}} \mathrm{d} \rightarrow \pi^{0} \eta$ and $\overline{\mathrm{p}} \mathrm{d} \rightarrow 3 \pi^{0} \eta$, are forbidden or rare in annihilation from the $\bar{p}$ p system in S-state. We conclude that the different shapes of the distributions for even and odd numbers of neutral particles are likely to be due to differences in the $\mathrm{S}$ - and P-capture probabilities. Also rescattering of pions off the spectator nucleon is likely to play an important role. However, there is no evidence for contributions from bound quasinuclear states.

In order to derive upper limits for the production of narrow states, we simulated by Monte Carlo a narrow (10 $\mathrm{MeV}$ ) resonance $\mathrm{N} \overline{\mathrm{N}}(1855)$ leading to a recoil momentum of $165 \mathrm{Mev} / \mathrm{c}$. We add the Monte Carlo spectrum to real data in Fig. 7. A fit identifies the resonance contribution clearly with $4 \sigma$ or more as long as the yield is larger than $5 \cdot 10^{-4}$. In the data we do not find a peak at this level. We conclude that no quasinuclear bound $\overline{\mathrm{N}} \mathrm{N}$ states were produced in any of the reactions, at least not with a yield exceeding $5 \cdot 10^{-4}$.

\section{Summary and conclusions}

$\overline{\mathrm{N}} \mathrm{N}$ potential models make a number of predictions which have not yet been thoroughly tested so far. In particular we searched for a $\bar{n} n$ component in the protonium wave function at annihilation which is predicted to de- velop when antiprotons and protons have atomic orbits with sufficient overlap to allow annihilation. A comparison of annihilation frequencies in $\mathrm{H}_{2}$ and $\mathrm{D}_{2}$ did not show evidence for this effect. $\overline{\mathrm{N}} \mathrm{N}$ annihilation into $\overline{\mathrm{K} K}$ as well as $\rho-\omega$ interference seem to favor an isospin decomposition of the protonium wave function into two components, $I=0$ and $I=1$, which are of equal strengths; their amplitudes are relatively real or at least have no large phase difference. While these two observation are not necessarily in direct conflict with $\overline{\mathrm{N}} \mathrm{N}$ potential models (since precise predictions have not yet been made) these observations are nevertheless easily understood in terms of SU(3) relations [40]. We also find no evidence for narrow quasinuclear bound states close to the $\overline{\mathrm{N}} \mathrm{N}$ threshold. This again is in conflict with predictions of $\overline{\mathrm{N}} \mathrm{N}$ potential models. We are

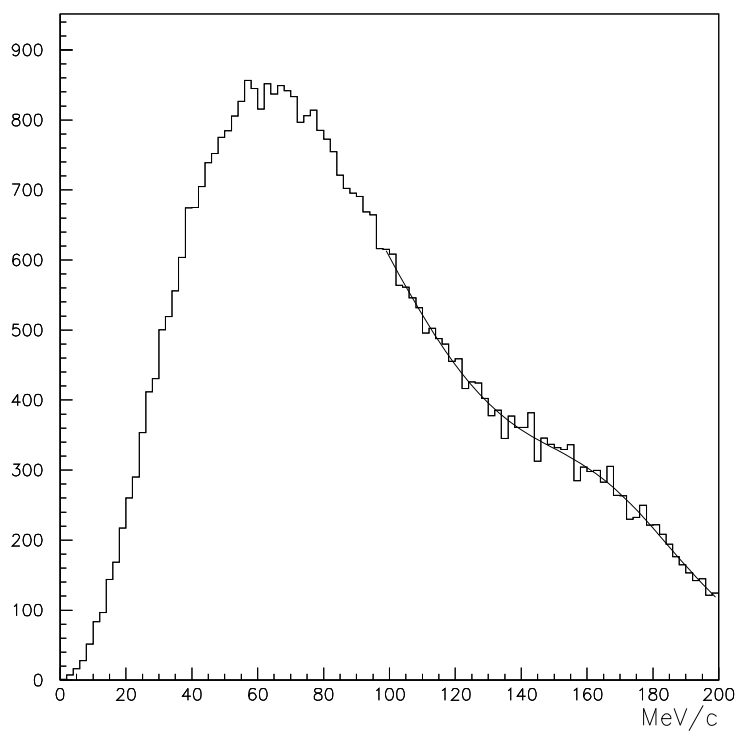

Fig. 6. Measured momentum distribution of $\overline{\mathrm{p}} \mathrm{d} \rightarrow 3 \pi^{0}+$ neutron with an additional $\overline{\mathrm{N}} \mathrm{N}$ (1855) resonance 
aware, however, that evidence for a $\overline{\mathrm{N}} \mathrm{N}$-like state at 2020 $\mathrm{MeV}$ was reported in several experiments; a summary is given in [44].

$\overline{\mathrm{N}} \mathrm{N}$ potential models were very popular at a time when the existence of narrow quasinuclear bound states and resonances had been claimed by many experiments; the models gave ample evidence that such states could exist and had even been predicted. When LEAR came into operation, the evidence for narrow states was quickly thrown into doubt. Scattering and annihilation were then the challanges which meson exchange models faced, often successfully as demonstrated in the calculation of the strong interaction shift and widths of $\bar{p} p$ atoms or in the successful determination of the pion nucleon coupling constant in the forward charge exchange reaction $\bar{p} p \rightarrow \bar{n} n$. But the success of $\overline{\mathrm{N}}$ potential models is certainly limited, and predictions have often failed. For example, the predicted isospin distortion of the protonium wave function due to pion exchange was not confirmed here. It seems to us that $\overline{\mathrm{N}} \mathrm{N}$ potentials govern the very peripheral part of $\overline{\mathrm{N}} \mathrm{N}$ interactions. Annihilation, on the other hand, is not a soft process. It does not belong to the realm where protons and antiprotons may be considered as fundamental particles.

We would like to thank the technical staff of the LEAR machine group and of all the participating institutions for their invaluable contributions to the success of the experiment. We acknowledge financial support from the German Bundesministerium für Bildung, Wissenschaft, Forschung und Technologie, the Schweizerischer Nationalfonds, the British Particle Physics andAstronomy Research Council, the U.S. Department of Energy and the National Science Research Fund Committee of Hungary (contractNo. DE-FG03-87ER40323, DE-AC03-76SF00098 DE-FGO2-87ER 40315 and OTKA F014357). N. Djaoshvili F.-H. Heinsius and K.M. Crowe acknowledge support from the A. von Humboldt Foundation.

\section{References}

1. R.A. Bryan and R.J.N. Phillips, Nucl. Phys. B (1968) 201

2. F. Bradamante and A. Martin, Phys. Lett. B343 (1995) 427

3. R. Timmermans, Th.A. Rijken and J.J. de Swart, Phys. Rev. C50 (1994) 48

4. J.M. Richard and M. Sainio, Phys. Lett. B110 (1982) 429

5. see C.J. Batty, Rep. Progr. Phys. 52 (1989) 1165 for a review

6. J. Carbonell et al., Z. Physik A334 (1989) 329

7. E. Klempt, Phys. Lett. B244 (1990) 122

8. I.S. Shapiro, Phys. Rep. C35 (1978) 129

9. W.W. Buck, C.B. Dover and J.-M. Richard, Ann. Phys. (N.Y.) 121 (1979) 71

10. L. Tauscher, in: Proc. $8^{\text {th }}$ European Symposium on Nucleon Antinucleon Interactions, Thessaloniki (Greece), 1986, ed. S. Charalambous et al., World Scientific, Singapore 1987, p. 247.
11. B. May et. al. (ASTERIX Collaboration), Phys. Lett. B225 (1989) 450; Z. Physik C46 (1990) 191, 203

12. C.B. Dover, Phys. Rev. Lett. 57 (1986) 1207

13. Crystal Barrel Collaboration, E. Aker et al., Nucl. Instrum. Meth. A 321 (1992) 69.

14. Crystal Barrel Collaboration, C. Amsler et al., Phys. Lett. B352 (1995) 187

15. Particle Data Group (PDG), Phys. Rev. D54 (1996) 1479.

16. Crystal Barrel Collaboration, A. Abele et al., Phys. Lett. B391 (1997) 191

17. Crystal Barrel Collaboration, C. Amsler et al., Z. Physik C58 (1993) 175

18. M. Gaspero, Physics of atomic nuclei 57 (1994) 1891 and references therein

19. D. Bridges et al., Phys. Rev. Lett. 56 (1986) 215

20. R. Bizzarri et al., Nucl. Phys. B690 (1974) 298

21. L. Gray et al., Phys. Rev. Lett. 30 (1973) 1091

22. D. Bridges et al., Phys. Lett. B180 (1986) 313

23. A. Angelopoulos et al., Phys. Lett. 212 (1988) 129

24. C. J. Batty, Proceedings of the LEAP98 Conference. To be published in Nucl. Phys. A.

25. C.J. Batty, Nucl. Phys. A601 (1996) 425

26. G. Reifenröther and E. Klempt, Phys. Lett. 245 (1990) 129

27. D.V. Bugg, unpublished

28. W.B. Kaufmann and H. Pilkuhn, Phys. Rev. C17 (1978) 215

29. Crystal Barrel Collaboration, C. Amsler et al., Phys. Lett. B411 (1997) 354

30. Crystal Barrel Collaboration, in preparation

31. H.B. O'Connell et al., Phys. Lett. B354 (1995) 14

32. Crystal Barrel Collaboration, C. Amsler et al., Phys. Lett. B346 (1995) 363

33. E. Klempt, Z. Physik A331 (1988) 211

34. C. Rossi and L. Montanet, Physics Reports 63 (1980) 149

35. W. Brückner et al., Phys. Lett. B158 (1986) 302; 166 (1986) 113; 169 (1986) 302

36. D.V. Bugg et al., Phys. Lett. B194 (1987) 563

37. T. Kalogeropoulos et al., Phys. Rev. Lett. 34 (1975) 1047

38. O.D. Dalkarov, contribution to LEAP 1996, Dinkelsbühl, Germany

39. L.N.Bogdanova, O.D.Dalkarov, I.S.Shapiro Annals of Physics 84, (1974) 261

40. E. Klempt, J.G. Korner and F. Walter, Z. Physik A354 (1996) 67

41. C. Amsler et al., Phys. Lett. B355 (1995) 425

42. S. Resag, Ph.D. thesis, University of Bonn (1996)

43. A. Abele et al., Phys. Lett. B380 (1996) 453

44. A. Ferrer et al., Eur. Phys. J. C10 (1999) 249 